Giulia Polo, Alessandro P. Burlina, Enzo Ranieri, Francesca Colucci, Laura Rubert, Antonia Pascarella, Giovanni Duro, Albina Tummolo, Andrea Padoan, Mario Plebani and Alberto B. Burlina*

\title{
Plasma and dried blood spot lysosphingolipids for the diagnosis of different sphingolipidoses: a comparative study
}

https://doi.org/10.1515/cclm-2018-1301

Received December 6, 2018; accepted April 14, 2019; previously published online May 15, 2019

\section{Abstract}

Background: Lysosphingolipids, the N-deacylated forms of sphingolipids, have been identified as potential biomarkers of several sphingolipidoses, such as Gaucher, Fabry, Krabbe and Niemann-Pick diseases and in GM1 and GM2 gangliosidoses. To date, different methods have been developed to measure various lysosphingolipids (LysoSLs) in plasma. Here, we present a novel liquid chromatography tandem mass spectrometry (LC-MS/MS) assay for a simultaneous quantification of LysoSLs (HexSph, LysoGb3, LysoGM1, LysoGM2, LysoSM and LysoSM509) in dried blood spot (DBS). This LC-MS/MS method was used to compare the levels of LysoSLs in DBS and plasma in both affected patients and healthy controls.

Methods: Lysosphingolipids were extracted from a $3.2 \mathrm{~mm}$ diameterDBSwithamixture of methanol:acetonitrile:water

\footnotetext{
*Corresponding author: Dr. Alberto B. Burlina, Division of Inherited Metabolic Diseases, Regional Center for Expanded Neonatal Screening, Department of Women and Children's Health, University Hospital of Padova, Via Orus 2/c, 35129 Padova, Italy, Phone: +39 0498217472, Fax: +39 0498217474,

E-mail: alberto.burlina@unipd.it

Giulia Polo, Francesca Colucci, Laura Rubert and Antonia

Pascarella: Division of Inherited Metabolic Diseases, Regional Center for Expanded Neonatal Screening, Department of Women and Children's Health, University Hospital of Padova, Padova, Italy Alessandro P. Burlina: Neurological Unit, St. Bassiano Hospital, Bassano del Grappa, Italy

Enzo Ranieri: Department of Biochemical Genetics, Directorate of Genetics and Molecular Pathology, SA Pathology, Women's and Children's Hospital, North Adelaide, South Australia, Australia Giovanni Duro: Institute of Biomedicine and Molecular Immunology (IBIM), National Research Council, Palermo, Italy

Albina Tummolo: Department of Metabolic Diseases, Clinical Genetics and Diabetology, Giovanni XXIII Children's Hospital, Bari, Italy Andrea Padoan and Mario Plebani: Department Laboratory Medicine, University Hospital of Padova, Padova, Italy. https://orcid.org/0000-0003-1284-7885 (A. Padoan)
}

(80:15:5, v/v) containing internal stable isotope standards. Chromatographic separation was performed using a C18 column with a gradient of water and acetonitrile both with $0.1 \%$ formic acid in a total run time of $4 \mathrm{~min}$. The compounds were detected in the positive ion mode electrospray ionization (ESI)-MS/MS by multiple reaction monitoring (MRM).

Results: The method was validated on DBS to demonstrate specificity, linearity, lowest limit of quantification, accuracy and precision. The reference ranges were determined in pediatric and adult populations. The elevated levels of LysoSLs were identified in Gaucher disease (HexSph), Fabry disease (LysoGb3), prosaposin deficiency (HexSph and LysoGb3) and Niemann-Pick disease types A/B and C (LysoSM and LysoSM509). The correlation in the levels between DBS and plasma was excellent for LysoGb3 and HexSph but poor for LysoSM and LysoSM509.

Conclusions: Despite the fact that plasma LysoSLs determination remains the gold standard, our LC-MS/MS method allows a rapid and reliable quantification of lysosphingolipids in DBS. The method is a useful tool for the diagnosis of different sphingolipidoses except for Niemann-Pick type C.

Keywords: biomarkers; dried blood spot (DBS); Fabry disease; gangliosidosis GM1; gangliosidosis GM2; Gaucher disease; Krabbe disease; Niemann-Pick type A/B disease; Niemann-Pick type C disease.

\section{Introduction}

Lysosomal storage disorders (LSD) are a group of 50 inherited inborn errors of metabolism characterized by a deficiency of a lysosomal enzyme. These LSDs have a broad spectrum of phenotypes that can lead to a delay in diagnosis. In some diseases, the delay of diagnostic procedure can negatively affect the efficacy of treatment, such as enzyme replacement therapy, substrate 
reduction or bone marrow transplantation. For the majority of these LSDs, the measurement of the specific enzyme activity in white cells (leukocytes and lymphocytes) is considered the gold standard for the diagnosis of these disorders. In 2001, Chamoles et al. introduced for the first time the use of the whole dried blood spot (DBS) to determine the enzymatic activity for several of LSDs [1] using fluorescence 4-methlyumbelliferone as the substrate. Subsequently, the use of a DBS for the enzymatic determination was proposed as an alternative for the rapid diagnosis of LSD. A DBS offers particular advantages over using conventional samples such as being easy to take, small blood volume, storage and transport conditions (room temperature) [2]. The enzymatic activity in DBS was recently measured by tandem mass spectrometry (MS/MS) [3, 4]. The versatility of MS/ MS has enabled this technique to be applied not only for enzymatic determination but also for biomarker detection [5]. In this context, lysosphingolipids (LysoSLs), the $\mathrm{N}$-deacylated forms of sphingolipids, have been identified as potential biomarkers of several LSDs, specifically the sphingolipidoses, such as Gaucher disease (GD), Fabry disease (FD), Krabbe disease (KD) and NiemannPick type A/B (NPA/B) and type C (NPC) diseases, and the GM1 and GM2 gangliosidoses. A number of studies showed the use of the measurement of LysoSLs in either plasma or DBS: lysoglobotriaosylceramide (Lyso-Gb3) for FD [6-10], galactosylsphingosine (GalSph, also called psychosine) for KD [11-13], glucosylsphingosine (GlcSph) for GD [14-17], lysosphingomyelin (LysoSM) for NPA/B $[18,19]$ and lysosphingomyelin 509 (LysoSM509) for NPC and NPAB [19].

We recently described a rapid liquid chromatography tandem mass spectrometry (LC-MS/MS) method for the plasma simultaneous quantification of HexSph (GalSph + GlcSph), LysoGb3 and LysoSM, including the LysoSM509 species [20]. Other studies confirmed the usefulness of a multiplex assay for LysoSLs in plasma as a reliable tool for the diagnosis of sphingolipidoses [21-23].

We developed and validated a new assay for the simultaneous determination of LysoSLs in DBS by MS/ MS. The concentration of LysoSLs was determined in healthy controls and in patients affected by the following sphingolipidoses: Fabry, Gaucher, Krabbe, NiemannPick types A, B and C diseases, prosaposine deficiency and GM1 and GM2 gangliosidoses. We also performed LysoSLs measurement in the DBS of neonates detected by neonatal screening to evaluate the interest of these biomarkers for the second-tier test of some lysosomal disorders.

\section{Materials and methods}

\section{Chemicals and reagents}

LC-MS-grade acetonitrile and methanol and high performance liquid chromatography (HPLC)-grade DMSO were purchased from Sigma Aldrich (St Louis, MO, USA). The UPLC-MS-grade formic acid (purity $>99 \%$ ) was supplied by Biosolve-Chemicals (Dieuze, France). Ultrapure water was generated using the Milli-Q system (Millipore, Bedford, MA, USA). The lipid standards 1-beta-D-glucosylsphingosine (GlcSph), lysoglobotriaosylsphingosine (LysoGb3), lyso-Sphingomyelin (LysoSM) and lyso-monosialoganglioside $\mathrm{GM}_{1}$ (LysoGM1) were bought from Matreya LLC (State College, PA, USA). Glucosyl(b)sphingosine-d5 (GlcSph-d5) and lyso-sphingomyelin-d7 (LysoSM-d7) were purchased from Avanti Polar Lipids and lyso-Monosialoganglioside $\mathrm{GM}_{2}$ (LysoGM2) from Takara Bio Inc. (Shiga, Japan).

\section{Patient samples}

The DBS samples were obtained from the patients referred to the Division of Inherited Metabolic Disease, University Hospital of Padua. The cohort included 114 pediatric and adult patients (mean age 37 years, range $0-77$ years) affected by GD type $1(n=25)$ GD type $3(n=1)$, FD ( $\mathrm{n}=36$ males, $\mathrm{n}=25$ females), KD $(\mathrm{n}=2)$, Niemann-Pick type A/B diseases (NPA/B, $n=6)$, Niemann-Pick type C (NPC, $n=4$, all NPC1), GM1 $(n=1)$ and GM2 $(n=2)$ gangliosidoses and prosaposin deficiency (PSAPD, $n=2$ ). Except for the five GD patients whose LysoSLs biomarkers were determined in newborn DBSs, all the other DBSs were collected during clinical monitoring. All of the patients had a confirmed diagnosis based on enzyme and molecular assay. To establish normal reference values for each biomarker, the control samples were taken from 253 anonymous healthy adult blood donors $(n=133)$ and pediatric patients $(n=120)$ with a collection range of $0-79$.

The DBSs were collected using standard procedures by puncturing the fingertip with a lancet and collecting the whole blood. Blood was dripped onto a 903 sample collection card (GE Healthcare, Cardiff, UK), which was air dried for approximately $2 \mathrm{~h}$. The samples collected as EDTA whole blood were spotted onto the filter paper in our laboratory using $50 \mu \mathrm{L}$ of whole blood.

The blood specimens used for the plasma to DBS method comparison were collected from healthy individuals with a confirmed LSD $(n=306)$. From each individual, blood was collected into an EDTA tube, and $50 \mu \mathrm{L}$ of the whole blood was spotted on filter paper. The remaining blood was centrifuged for $10 \mathrm{~min}$ at $2000 \mathrm{~g}$, and the plasma was stored at $-80{ }^{\circ} \mathrm{C}$. All of the DBSs were subsequently stored at $-20{ }^{\circ} \mathrm{C}$ in zip-lock plastic bags containing desiccant. Ethical approval was obtained from the Ethics Committee of Padua Hospital. LysoSLs concentrations in the plasma were measured by LC-MS/MS using the method recently described [20].

\section{DBS calibration standards and quality control (QC) preparation}

The stock standard solutions and working solutions for all analytes were prepared gravimetrically as described earlier by Polo et al. [20]. GlcSph was used as the HexSph standard for the calibration 
curve and quality controls. The stock standard solutions of LysoGM1 and LysoGM2 were prepared by weight with a final concentration of $0.1 \mathrm{mg} / \mathrm{mL}$. The extraction solution with internal standards was prepared by diluting in a solution of methanol:acetonitrile:water (80/15/5, v/v/v) with LysoSM-d7 and GlcSph-d5 at $2.5 \mathrm{nmol} / \mathrm{L}$ and stored at $-80^{\circ} \mathrm{C}$ before use.

An eight-level calibration curve was prepared by spiking the pure standards with pooled human blood prior to spotting (range: 0-100 nmol/L for LysoGb3, LysoGM1 and LysoGM2; 0-1000 nmol/L for LysoSM and HexSph). For the measurement of LysoSM-509, we assumed a relative response ratio of 1 to LysoSM (note that a commercial standard is not yet available). The precision and accuracy of the assay were evaluated using the QCs at three concentration levels, which were prepared from pooled EDTA blood. For LysoGb3, LysoGM1 and LysoGM2, QC1 endogenous, QC2 endogenous $+8 \mathrm{nmol} / \mathrm{L}$ and QC3 endogenous $+80 \mathrm{nmol} / \mathrm{L}$ were used. For LysoSM and HexSph, QC1 endogenous, QC2 endogenous $+80 \mathrm{nmol} / \mathrm{L}$ and QC3 endogenous $+800 \mathrm{nmol} / \mathrm{L}$ were used. About $50 \mu \mathrm{L}$ of spiked blood was then spotted onto the 903 sample collection card, dried at room temperature and stored at $-20^{\circ} \mathrm{C}$ until analysis.

\section{Sample preparation}

To extract lysosphingolipids from DBS, a 3.2-mm disc was punched into a 96-well microplate, and $100 \mu \mathrm{L}$ of extraction solution was added. The microplate was incubated for $1 \mathrm{~h}$ at $45^{\circ} \mathrm{C}$ with orbital shaking (500 rpm). After incubation, $50 \mu \mathrm{L}$ water Milli-Q was added, and the diluted extracted mixture was transferred to a new microplate. Then, $8 \mu \mathrm{L}$ was injected in the LC-MS/MS system.

\section{LC-MS/MS}

The lysosphingolipid measurements were performed by reversephase liquid chromatography using the Waters Acquity UPLC and the BEH C18 column $2.1 \times 50 \mathrm{~mm}$ with a $1.7 \mu \mathrm{m}$ particle size (Waters, USA). Mass spectrometry detection was conducted with a Xevo TQs micro-detector (Waters, USA) set in the positive mode using an electrospray ionization source (ESI). LC-MS/MS analysis was performed as previously described [20] with the inclusion of the multiple reaction monitoring (MRM) transitions of GlcSphd5, LysoSM-d7, LysoGM1 and LysoGM2. The two MRM transitions selected for LysoGM1 and LysoGM2 were 1118.73-204.06 m/ $z$ and 1280.65-204.07 m/z with CE $40 \mathrm{~V}$ and CE $56 \mathrm{~V}$, respectively. The $\mathrm{CV}$ was $30 \mathrm{~V}$ for both. All of the parameters were optimized by the direct infusion of standard solutions into mass spectrometry to obtain a better signal.

\section{Method validation}

The accuracy of the method was assessed by performing recovery studies using the QCs. Accuracy was given by percent relative error (\%RE) and calculated by subtracting the nominal level from the mean amount divided by the theoretical amount and then multiplied by 100: ([mean-nominal]/[nominal] $\times 100)$. Within-run and between-run precision were determined by preparing and analyzing each QC 10 times per run over 10 consecutive working days. The limit of detection (LOD) for each biomarker was determined using a signal-to-noise ratio of 3. Peaks with a signal-to-noise ratio greater than 10 were accepted and integrated (lower limit of quantification).

\section{Data analysis}

Statistical analysis was performed using GraphPad Prism software (GraphPad Software Inc., San Diego, CA, USA) and MedCalc. The LysoSL control values were expressed as the median and the 2.5th-97.5th percentiles of the distributions. The LysoSL values for the LSD-affected patients were expressed as the median with minimum/maximum values because of the small number of samples for each disease. Deming's regression procedure and the Bland-Altman analysis were used to compare the plasma and DBS methods.

\section{Results}

\section{DBS method validation}

The LysoGb3, LysoSM, HexSph (GalSph+GlcSph), LysoGM1 and LysoGM2 standards were easily detectable by MS/MS (Supplementary material, Figure 1). The deuterated internal standards of LysoSM (LysoSM-d7) and GlcSph (GlcSph-d5) were used instead of GlcSph from a plant to correct for extraction efficiency and any matrix effects (Table 1). The linearity studies, lower LOD and limit of quantification are given in Table 2. The correlation coefficients $\left(R^{2}\right)$ were acceptable at 0.995 except those for LysoGM1 and LysoGM2 at greater than 0.97. The higher point of the calibration curve (high RSD) was excluded. For both analytes, the DBS gave lower linearity performance that what was obtained for plasma or aqueous solutions. The endogenous levels of LysoGb3, HexSph and LysoSM were calculated from the y-intercept concentrations for the multiple calibration curves $(n=3)$ except those for LysoGM1 and LysoGM2, which were undetectable in the pooled whole blood.

The intra-assay precision of LysoGb3, LysoSM and HexSph was better than $10 \%$ at all levels. For both LysoGM1 and LysoGM2, the intra-assay precision exceeded $15 \%$ and $20 \%$, respectively (Table 3 ). The interassay precision was below $20 \%$ for all analytes except LysoGM1 and LysoGM2 (medium level: 33.2\% and 24.4\%, respectively). Carryover was determined by injecting a blank sample after the highest calibration standard and finding it negligible. 
Table 1: MRM parameters for the quantification of LysoSLs and the corresponding internal standard.

\begin{tabular}{|c|c|c|c|c|c|c|}
\hline Lysosphingolipids & $\begin{array}{r}\text { Parent, } \\
m / z\end{array}$ & $\begin{array}{r}\text { Daughter, } \\
m / \mathbf{z}\end{array}$ & $\begin{array}{r}\text { Cone } \\
\text { voltage, } \mathrm{V}\end{array}$ & $\begin{array}{l}\text { Collision } \\
\text { energy, V }\end{array}$ & $\begin{array}{c}\text { Retention } \\
\text { time, min }\end{array}$ & $\begin{array}{l}\text { Internal } \\
\text { standard }\end{array}$ \\
\hline LysoGb3 & 786.5 & 282.3 & 20 & 35 & 1.69 & GlcSph-d5 \\
\hline HexSph & 462.3 & 282.3 & 30 & 20 & 1.90 & GlcSph-d5 \\
\hline GlcSph-d5 & 467.3 & 287.3 & 30 & 20 & 1.89 & - \\
\hline LysoSM & 465.4 & 184.1 & 30 & 22 & 1.74 & LysoSM-d7 \\
\hline LysoSM-d7 & 472.5 & 184.1 & 30 & 22 & 1.73 & - \\
\hline LysoSM-509 & 509.0 & 184.1 & 30 & 22 & 2.52 & LysoSM-d7 \\
\hline LysoGM1 & 1280.6 & 204.1 & 20 & 56 & 1.86 & GlcSph-d5 \\
\hline LysoGM2 & 1118.6 & 204.1 & 20 & 45 & 1.92 & GlcSph-d5 \\
\hline
\end{tabular}

Mass spectrometer: Waters Xevo TQS micro.

Table 2: Validation data of LysoSLs: range of measurement and linearity.

\begin{tabular}{lrrrr}
\hline & $\begin{array}{r}\text { Linearity, } \\
\mathbf{R}^{\mathbf{2}}(\mathbf{n}=\mathbf{3})\end{array}$ & $\begin{array}{r}\text { LLOD, } \\
\mathbf{n m o l} / \mathbf{L}\end{array}$ & $\begin{array}{r}\text { LLOQ, } \\
\mathbf{n m o l} / \mathbf{L}\end{array}$ & $\begin{array}{r}\text { Upper limit } \\
\text { of linearity }\end{array}$ \\
\hline LysoGb3 & 0.995 & 0.26 & 0.45 & 197 \\
LysoSM & 0.994 & 5.15 & 10.31 & 2121 \\
HexSph & 0.996 & 0.81 & 1.62 & 1965 \\
LysoGM1 & 0.979 & 2.50 & 5 & 105.1 \\
LysoGM2 & 0.975 & 1.25 & 2.5 & 106.9 \\
\hline
\end{tabular}

\section{Lysosphingolipids in DBS samples}

Table 3 presents the DBS levels of LysoGb3, HexSph, LysoSM, LysoSM509, LysoGM1 and LysoGM2 in the control and in patients with LSD.
In the unaffected controls, the concentrations of the metabolites LysoGb3, HexSph, LysoSM and LysoSM509 were low but within the method detectable limit, and those of LysoGM1 and LysoGM2 were undetectable. No measurable differences were found in the levels of these metabolites by sex or age.

The MS/MS multiplex method was applied to determine the levels of LysoSLs in the 114 patients affected by LSD (Table 3 and Figure 1). The levels of LysoSLs identified in each sphingolipidosis had a specific pattern.

LysoGb3 was increased in all FD patients. The levels were higher in males with the classical form of the disease and were at the intermediate level in males with lateonset form and in females (Figure 1A). LysoGb3 was also elevated albeit marginally in GD and in two patients with prosaposin deficiency.

Table 3: Analytical performance of the LC-MS/MS method: intra-day and inter-day precision (percent coefficient of variation) at three concentration levels and accuracy (percent relative error).

\begin{tabular}{|c|c|c|c|c|c|c|c|c|}
\hline & \multicolumn{2}{|l|}{ QC1 (low) } & \multicolumn{3}{|c|}{ QC2 (medium) } & \multicolumn{3}{|r|}{ QC3 (high) } \\
\hline & $\begin{array}{l}\text { Mean } \pm S D \\
\text { nmol/L }\end{array}$ & $\begin{array}{r}\text { Precision, } \\
\text { CV\% }\end{array}$ & $\begin{array}{r}\text { Mean } \pm \mathrm{SD} \\
\mathrm{nmol} / \mathrm{L}\end{array}$ & $\begin{array}{r}\text { Precision, } \\
\text { CV\% }\end{array}$ & $\begin{array}{r}\text { Accuracy, } \\
\text { RE\% }\end{array}$ & $\begin{array}{r}\text { Mean } \pm \mathrm{SD} \\
\mathrm{nmol} / \mathrm{L}\end{array}$ & $\begin{array}{r}\text { Precision, } \\
\text { CV\% }\end{array}$ & $\begin{array}{r}\text { Accuracy, } \\
\text { RE\% }\end{array}$ \\
\hline \multicolumn{9}{|c|}{ Intra-assay $(n=15)$} \\
\hline LysoGb3 & $1.74 \pm 0.17$ & 9.5 & $9.0 \pm 0.7$ & 7.7 & 90.7 & $62.2 \pm 4.4$ & 7.1 & 75.6 \\
\hline LysoSM & $157.8 \pm 9.3$ & 5.9 & $247.7 \pm 16.9$ & 6.8 & 112.5 & $1042.3 \pm 66.7$ & 6.4 & 110.6 \\
\hline HexSph & $20.2 \pm 0.9$ & 4.6 & $112.5 \pm 8.5$ & 7.6 & 115.4 & $889.9 \pm 57.0$ & 6.4 & 108.7 \\
\hline LysoGM1 & ND & & $7.8 \pm 0.9$ & 11.4 & 97.7 & $73.8 \pm 11.5$ & 15.5 & 92.3 \\
\hline LysoGM2 & ND & & $8.4 \pm 0.8$ & 10.0 & 104.7 & $95.6 \pm 6.9$ & 7.2 & 119.5 \\
\hline \multicolumn{9}{|c|}{ Inter-assay $(\mathrm{n}=15)$} \\
\hline LysoGb3 & $1.70 \pm 0.25$ & 14.6 & $10.2 \pm 1.4$ & 13.8 & 106.1 & $65.5 \pm 8.70$ & 13.3 & 79.8 \\
\hline LysoSM & $163.4 \pm 17.6$ & 10.8 & $252.0 \pm 35.6$ & 14.1 & 110.8 & $990.0 \pm 104.0$ & 10.6 & 103.3 \\
\hline HexSph & $20.5 \pm 2.4$ & 11.8 & $108.3 \pm 10.1$ & 9.3 & 109.8 & $850.2 \pm 69.6$ & 8.2 & 103.7 \\
\hline LysoGM1 & ND & & $7.1 \pm 2.3$ & 33.2 & 85.5 & $86.4 \pm 13.3$ & 15.4 & 108.0 \\
\hline LysoGM2 & ND & & $6.5 \pm 1.6$ & 24.4 & 81.3 & $89.6 \pm 16.8$ & 18.7 & 112.0 \\
\hline
\end{tabular}

$\mathrm{CV} \%$, percentage coefficient of variation; RE\%, percentage relative error; SD, standard deviation; ND, not detectable. 
A

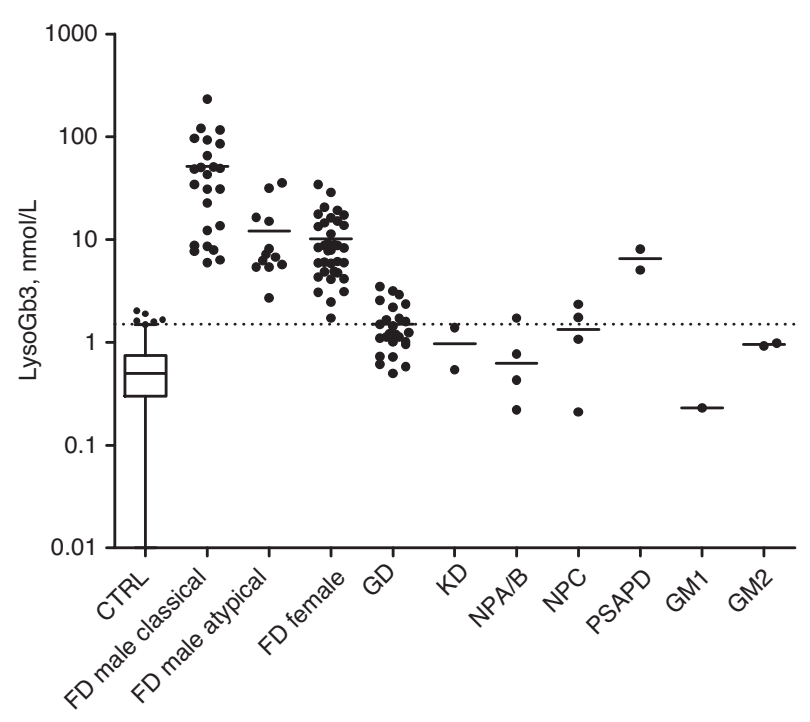

C

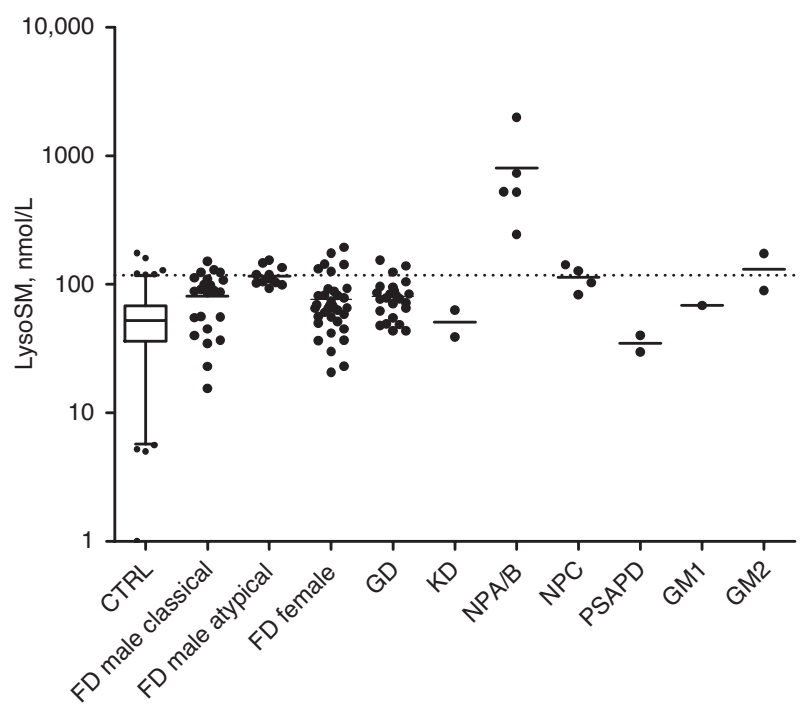

B

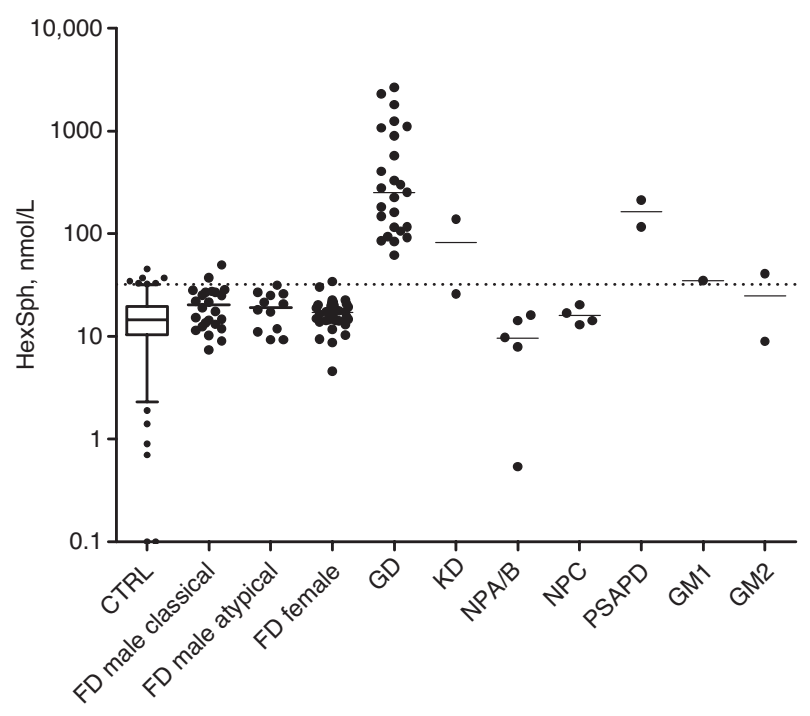

D

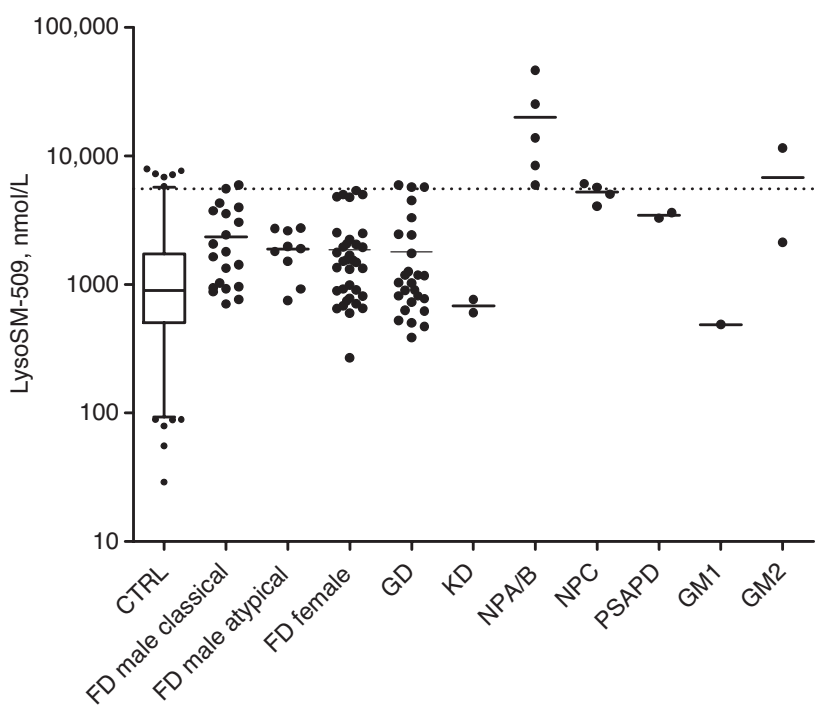

Figure 1: LysoSLs concentrations in the healthy controls (CTRL) and in patients with sphingolipidoses. LysoGb3 (A), HexSph (B), LysoSM (C) and LysoSM509 (D). Data in healthy controls are represented as box 25-75th percentile (whiskers: 2.5th and 97.5th), in affected patients as scatter plots. Horizontal lines indicate the median of the distribution. Horizontal dashed lines represent the 97.5 th percentile of the healthy population. All graphs are depicted in the ${ }^{10} \log$ scale.

A 17-fold elevation in HexSph was observed in the DBS from the GD patients vs. the controls (Figure 1B). The analysis of the neonatal DBS of 5 GD patients identified by newborn screening revealed a significant elevation of HexSph (mean: $113.0 \mathrm{nmol} / \mathrm{L}$, range: $92-163.8 \mathrm{nmol} / \mathrm{L}$, healthy controls $2.3-31.1 \mathrm{nmol} / \mathrm{L}$ ) (Supplementary material, Table S1). In KD, HexSph was moderately increased in one early infantile KD patient but not in the other asymptomatic KD patients (sample collected on the fifth day of life in siblings of the infantile KD patient).
LysoSM was clearly increased in patients with NPA/B by a factor of 10 (Figure 1C). A significant but less pronounced increase was observed in the NPC $(p=0.002)$ and GD patients $(\mathrm{p}<0.0001)$, in which the levels were progressively reduced in comparison with the NPA/B patients. LysoSM509 showed a similar pattern with very high values in NPA/B and overlapping values with the upper range of unaffected controls in NPC and GD (Figure1D). LysoGM1 and LysoGM2 were below the LOD in the controls and in patients affected by sphingolipidoses 
other than GM1 and GM2 gangliosidoses (Figure 2). LysoGM1 was elevated in the patient with infantile GM1 gangliosidosis. LysoGM2 was detected in one patient with infantile Sandhoff disease but not in the adult Sandhoff patient (Table 3).

\section{Comparison between plasma and DBS}

The levels of LysoGb3, GlcSPh, LysoSM and LysoSM509 were compared among the specimen matrix types, DBS and plasma in the matched samples of unaffected controls and positive patients (Figure 3).

A linear correlation was observed between LysoGb3 in plasma and that in DBS in 298 cases $(y=0.35+0.945 \times$, $n=298, r^{2}$ 0.977). The plot given in Figure 3B shows the absence of a bias between the two matrix types.

For HexSph, a good linear correlation was observed (equation $\mathrm{y}=16.5354+2.7070 \times, \mathrm{n}=307, \mathrm{r}^{2}$ 0.970) between the two matrix types. A significant difference was observed between the two matrix types given by the slope (2.7) of the correlation, with the levels in DBS being higher than those in plasma with a mean bias of 134\% (95\% CI 42.5-225.6) (Figure 3D).

The comparison between plasma and DBS showed a poor correlation result for LysoSM within the full concentration range ( $0-856 \mathrm{nmol} / \mathrm{L}$ in the plasma, $\left.\mathrm{r}^{2} 0.816\right)$. A correlation between the values was observed at levels greater than $50 \mathrm{nmol} / \mathrm{L}$ in the plasma. The mean concentrations were higher in DBS than in the plasma in the values below $50 \mathrm{nmol} / \mathrm{L}$ in the plasma, and the DBS levels were higher than the plasma in the values above $50 \mathrm{nmol} / \mathrm{L}$.

A similar pattern was observed in LysoSM509 with a Pearson's $r$ of $0.5989(n=264)$ for the full range of concentration (0-10,000 $\mathrm{nmol} / \mathrm{L}$ in plasma). No correlation was found for data in $0-800 \mathrm{nmol} / \mathrm{L}$ in the plasma (Pearson's $r=-0.11 ; n=241$ ), and Pearson's $r$ was 0.84 for the values above $800 \mathrm{nmol} / \mathrm{L}$ in the plasma.

We compared the ability of LysoSLs levels to identify the affected patients in both matrixes of DBS and plasma (Figure 4). A receiver-operating characteristics (ROC) curve comparison was used for LysoGb3 (Figure 4A) and HexSph (Figure 4B) using the paired samples of the plasma and DBS from the patients affected by FD and GD disease, respectively, and then plotted against the unaffected or other sphingolipidoses. Generally, the two matrixes showed excellent performance with an AUC close to 1, but for both LysoSLs, the AUC was slightly higher in the plasma than in the DBS.

The comparison of LysoSM and LysoSM509 in the two matrixes was limited because of the number of available patients affected by NPA/B and NPC and the recourse of plotting the data graphically (Figure 5). In the plasma, the simultaneous determination of LysoSM and LysoSM509 was clearly able to distinguish between NPA/B from NPC and in the unaffected controls (Figure 5A). In the DBS, this separation was less pronounced, and only NPA/B patients showed an increase in LysoSM and
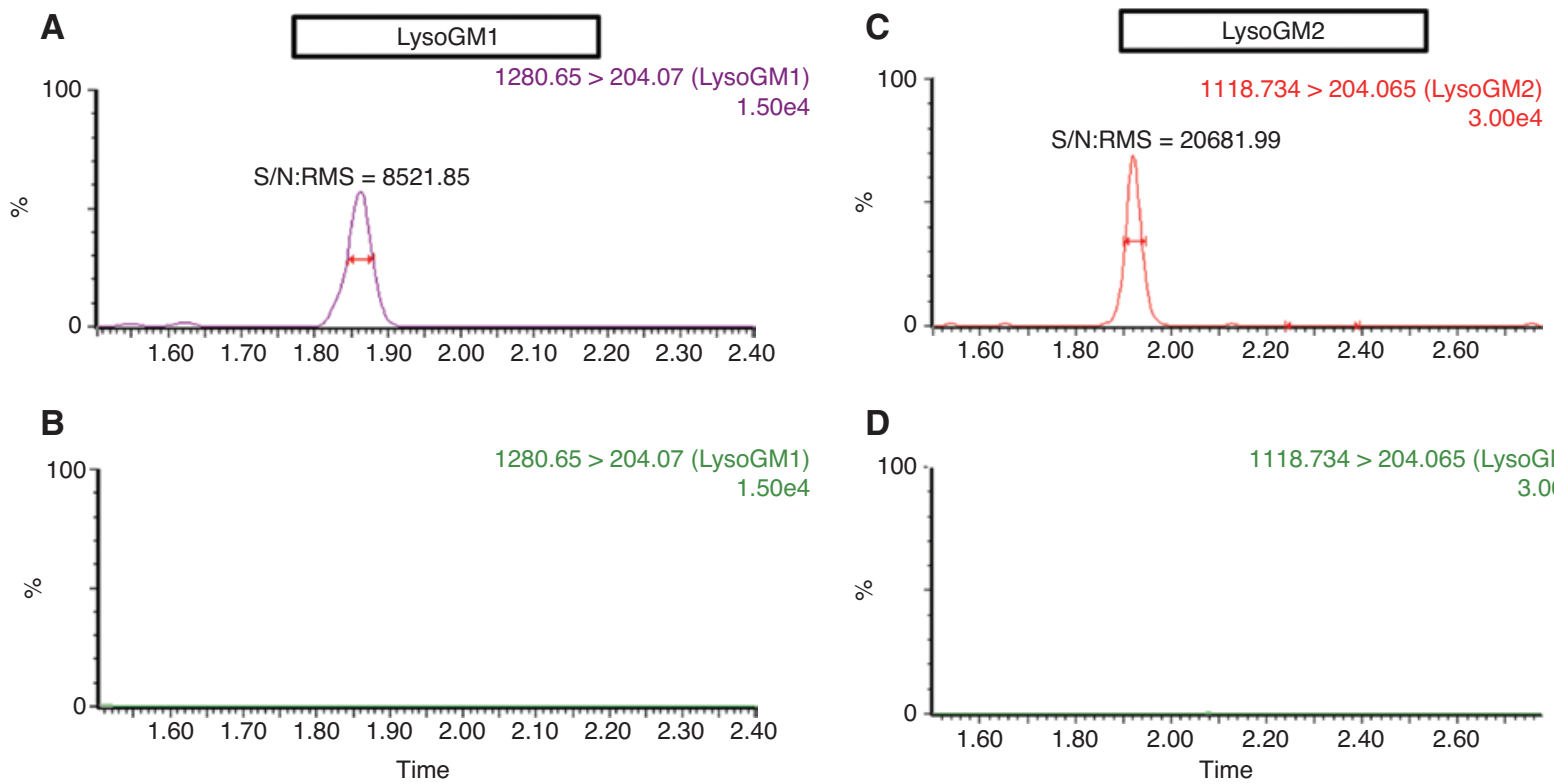

D

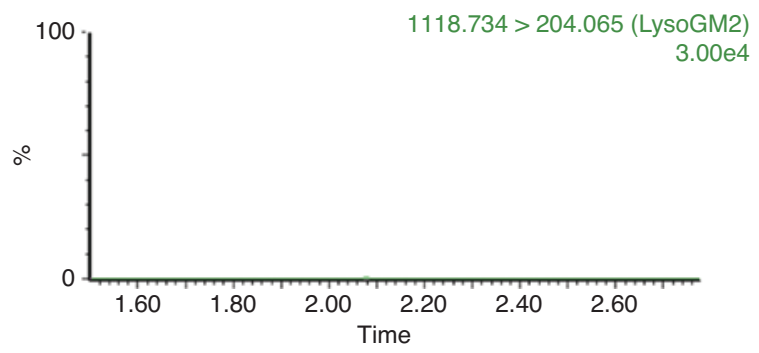

Figure 2: Chromatograms of LysoGM1 (A, B) and LysoGM2 (C, D). MRM transitions of LysoGM1 and LysoGM2 in GM1 gangliosidosis (A) and GM2 gangliosidosis (C), respectively. No signals are detected in the healthy controls (B for LysoGM1, D for LysoGM2). S/N, signal to noise; RMS, root mean square. 
A

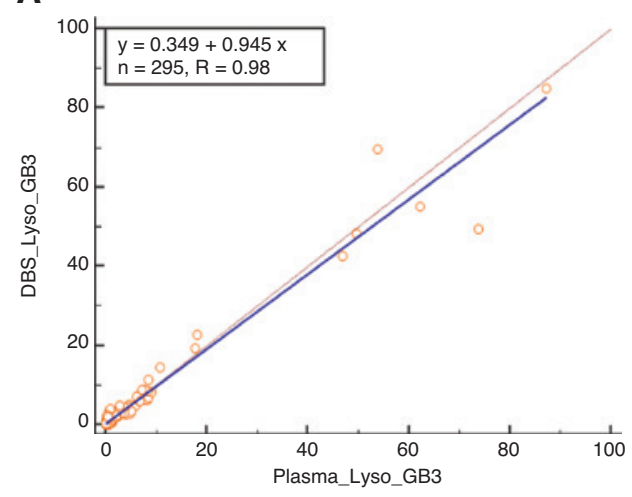

C

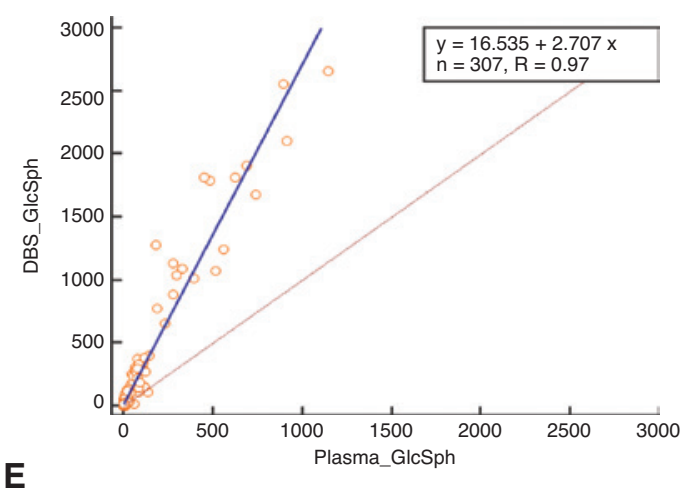

E
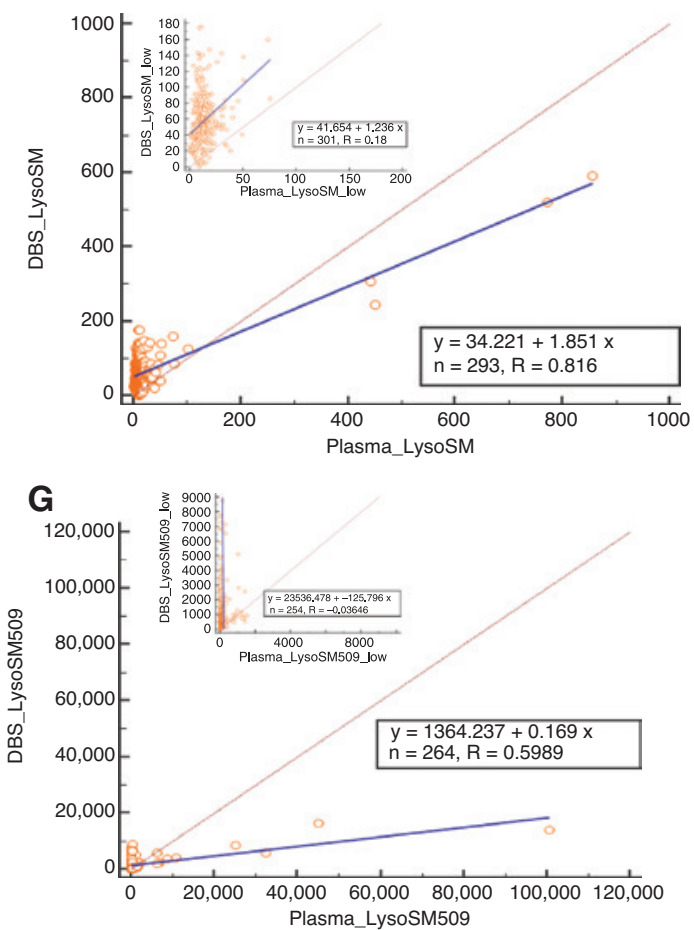

B

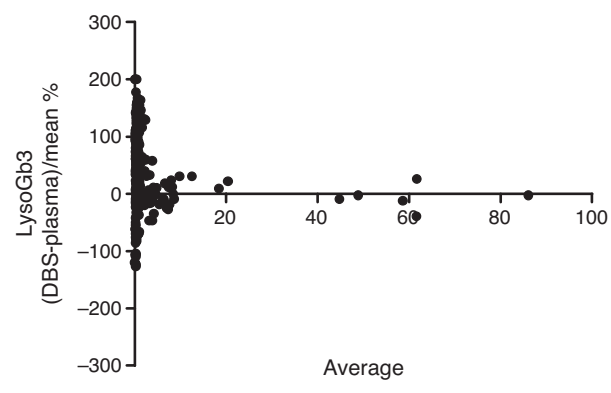

D

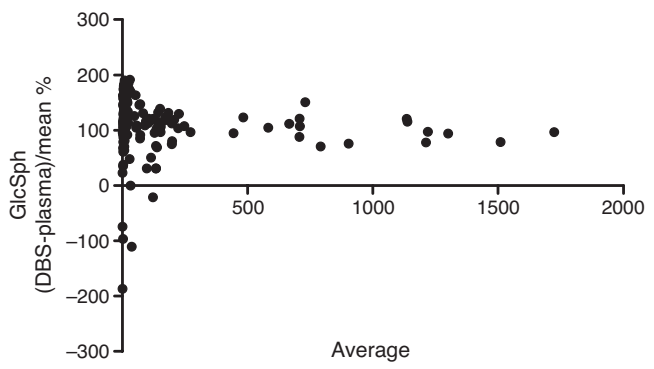

F

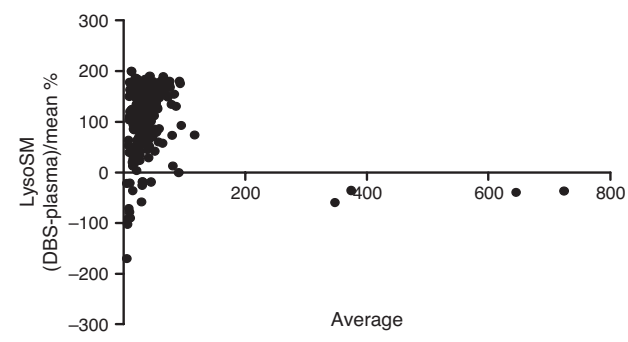

H

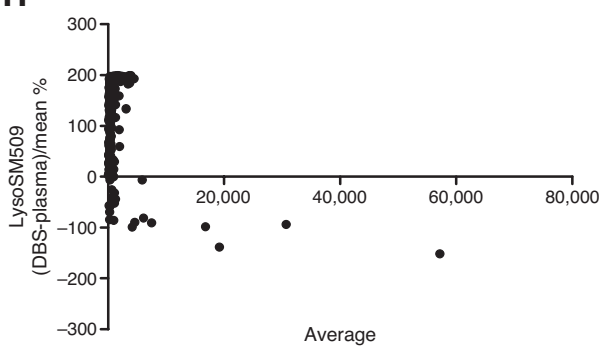

Figure 3: Comparison of the levels of LysoGb3 (A, B), HexSph (GlcSph) (C, D), LysoSM (E, F) and LysoSM509 (G, H) in the DBS and the plasma. The results obtained from both matrix were quantitatively compared using Deming's regression (left) $(y=D B S$ results; $x=$ plasma results; $\mathrm{nmol} / \mathrm{L}$ ). For LysoSM (E) and LysoSM509 (G), the full-range data are displayed in the full-sized figure, and the lower data points (for LysoSM, 0-100 nmol/L in the plasma; for LysoSM509, 0-1000 nmol/L in the plasma) are displayed as an inset in the graph. The determined slope, $y$-intercept and Pearson's r coefficient ( $r$ ) are shown. Bias was analyzed using the Bland-Altman plot (right) in which the $y$ axis is the value ([DBS-Plasma]/mean)\% vs. the average of the two methods. 

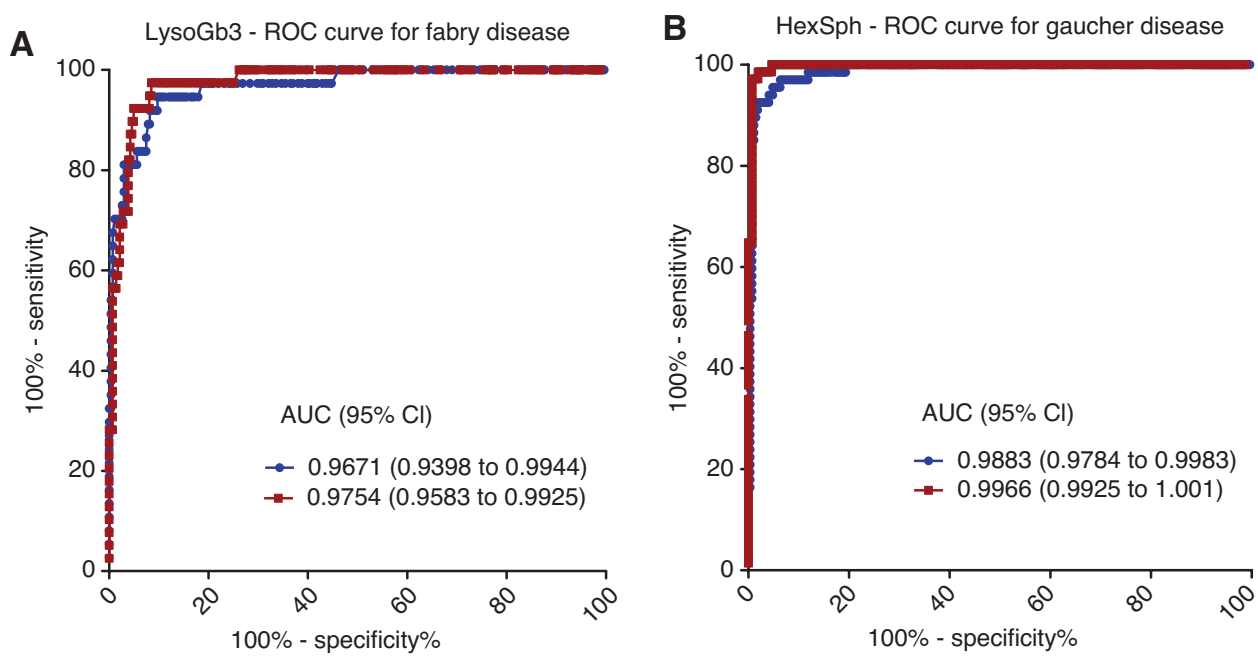

Figure 4: ROC analysis comparison between the plasma (red) and the DBS (blue) for LysoGb3 (A) and HexSph (B) for the Fabry and Gaucher diseases, respectively, compared to the healthy control group.

AUC, area under the curve; $95 \% \mathrm{Cl}, 95 \%$ confidence interval.
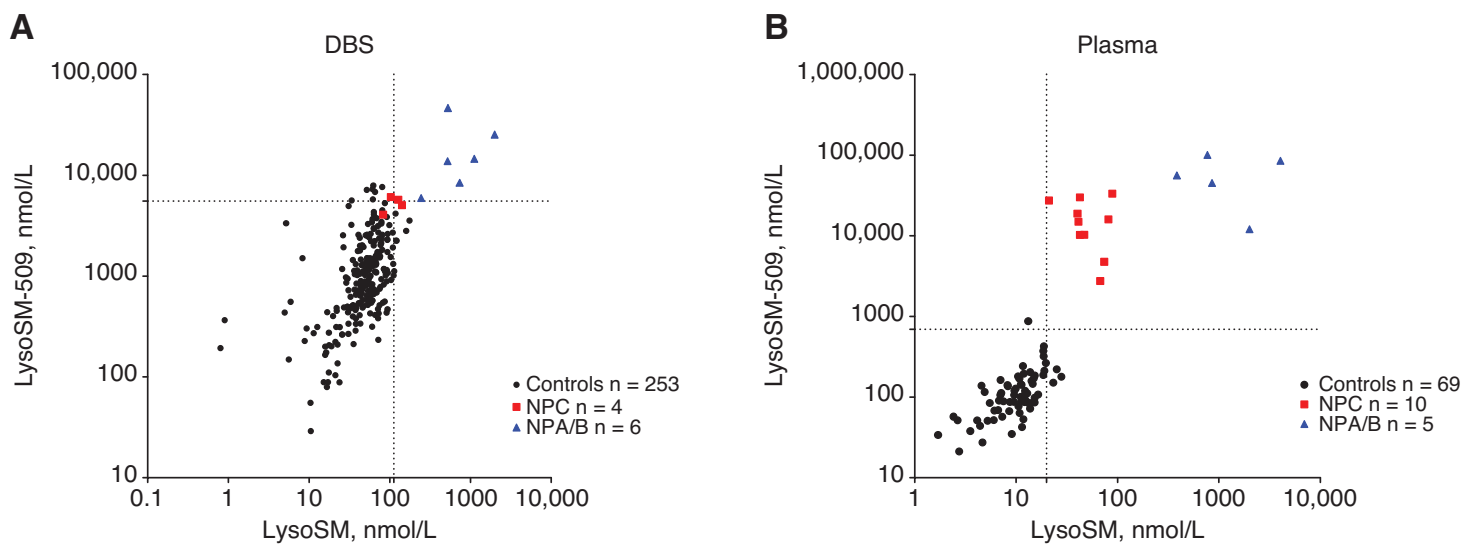

Figure 5: LysoSM and LysoSM509 levels (nmol/L) in the NPA/B and NPC patients measured in the DBS (A) and in the plasma (B). Discontinued lines indicate the limit of control values (97.5th percentile).

LysoSM509. In the NPC, the levels of both analytes were around the 95th percentile of the unaffected population (Figure 5A).

\section{Discussion}

Several biochemical markers have been recently identified that could assist in the disease diagnosis of several sphingolipidoses. We reported a sensitive multiplex method to measure various lysosphingolipids in the plasma for the diagnosis of Fabry, Gaucher, Krabbe, Niemann-Pick type A, B and C diseases and prosaposin deficiency [20, 24]. Similar studies were performed by other groups [5, 21-23]. Despite the different analytical approaches in terms of sample preparation, internal standard and LC-MS/MS analysis, all these studies confirmed that a simultaneous LysoSL quantification could be a useful first-tier test to screen patients suspected for sphingolipidoses.

In this study, we extended the simultaneous determination and quantified the LysoSLs levels in two sample matrix types, DBS and plasma, using the deuterated forms of LysoSM and GlcSph as the internal standard. We also included LysoGM1 and LysoGM2 in the panel for the detection of GM1 and GM2 gangliosidoses, respectively. Comparing the plasma with the DBS provided several advantages, namely, relatively non-invasive sampling, low cost sample collection and minimal pre-treatment requirements for sample processing and shipment.

Moreover, the quantification of the LysoSLs levels was performed by ultra-(U)HPLC-MS/MS from a small 
volume of sample, a 3-mm punch ( $\approx 3.2 \mu \mathrm{L}$ of blood), which provided a good analytical performance for LysoGb3, LysoSM and HexSph in terms of precision, accuracy and linearity. The analytical inter- and intra-assay performances were acceptable and highly reproducible with a coefficient of variation below 10\% (Table 3). LysoGM1 and LysoGM2 presented low ionization efficiency by MS/ MS with the highest signal-to-noise ratio achieved in the positive mode. Some quantitative limitations were observed for these two analytes as found in other studies performed in the plasma [21]. The concentrations were reported as a rough estimate (Table 4) partly because of the lack of a suitable isotopically labeled standard [19].

In this study, we quantified the LysoSLs levels in a large cohort of DBSs collected from unaffected controls $(n=253)$ and in patients affected by sphingolipidoses $(n=114)$. In general, the DBSs could clearly discriminate the controls from positive patients, consistent with the studies that used plasma [20,21]. We also compared the LysoSLs (LysoGb3, HexSph, LysoSM and LysoSM509) in the plasma and DBSs to determine the ability to identify affected patients between the two matrix types.

LysoGb3 in the DBSs was studied in a large cohort of Fabry patients $(n=71)$ composed of affected males with classic and late-onset phenotype and females. We observed a high elevation of LysoGb3 in males with the classic phenotype and a mild-to-moderate elevation in both males with the late-onset and heterozygous females. As previous studies suggested, a normal LysoGb3 could not exclude a Fabry disease in females but could very unlikely make a diagnosis of FD in men $[25,26]$. The amount of sample in a 3.2-mm blood spot was approximately 50 times less than that routinely used in the plasma, and both the sensitivity and specificity of LysoGb3 testing on DBS were only marginally reduced in comparison with those on the plasma (AUC DBS 0.9671 vs. AUC plasma 0.9754).

HexSph in the DBS of GD patients was highly elevated and consistent with the previous studies on plasma [14, 20]. The sensitivity of HexSph for GD in the DBS was highly satisfactory and only marginally less than that in the plasma (AUC DBS 0.9883 vs. AUC plasma 0.9966). A slight elevation of LysoGb3 was also observed in these patients [20, 21, 23].

HexSph has been reported to be moderately elevated in the plasma and DBS in cases of infantile $\mathrm{KD}[11,12,20]$. In this study, LysoSLs was tested in only two KD patients and only one patient with the symptomatic early infantile onset form had a high level of HexSph.

Two patients with PSAP deficiency (an ultra-rare disease), previously diagnosed by plasma LysoSLs testing [24], showed increased levels of LysoGb3 and HexSph in

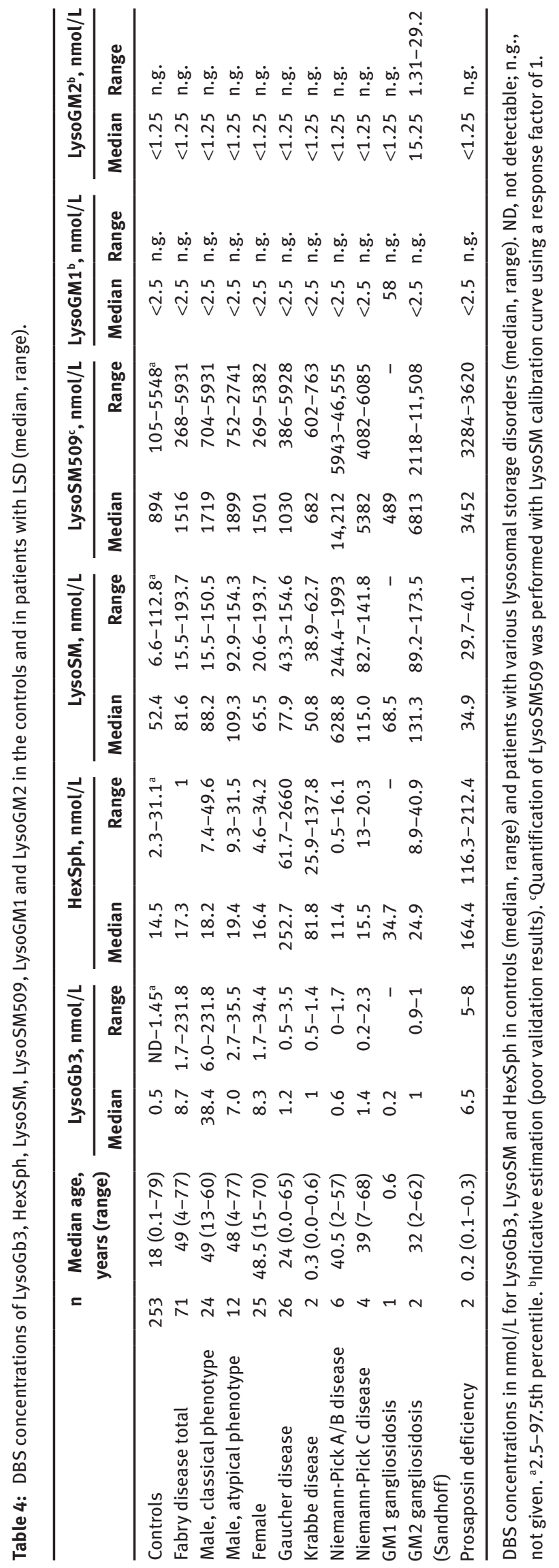


the DBS. To date, LysoSLs dosage is the only biochemical test available for saposin defects, both saposin C deficiency and prosaposin deficiency [21, 24].

With our method, the concentrations in both the plasma and DBS of LysoGb3 were highly correlated and able to identify the affected patients [27]. Similar to LysoGb3, a strong correlation was observed for HexSph between the plasma and DBS. For this metabolite, the plasma concentration could be estimated from DBS using a conversion factor of 2.7. The bias was not observed between the plasma and dried plasma spot (data not shown), and it could be explained in a different distribution of HexSph among the blood components (plasma and red cells).

LysoGM1 and LysoGM2 have been proposed to be biomarkers of other two ultra-rare lysosomal diseases, the GM1 and GM2 gangliosidoses. The sensitivity of these markers in the plasma has been reported to be poor and critical for later onset forms [5, 21]. We analyzed these markers in a limited number of patients $(\mathrm{GM} 1 \mathrm{n}=1 ; \mathrm{GM} 2 \mathrm{n}=2)$. Both the plasma and the DBS gave a normal LysoGM2 for patients with the late-onset form of GM2 called Sandhoff disease [28]. Both the GM1 and GM2 infantile forms were correctly identified in the DBS.

An increase in the plasma levels of both LysoSM and LysoSM509 in the plasma is the hallmark of NPA/B and NPC diseases [19, 29, 30]. The combined determination of these metabolites in the plasma allowed the discrimination between the two disorders [5, 31]. In the DBS from NPB patients, LysoSM was found to be elevated approximately five-fold and did not overlap with the normal controls, thus making it a potentially useful biomarker [18]. Earlier studies suggested that the analyses of LysoSM and LysoSM509 in DBS samples were inferior to the measurements in the plasma and should be performed only in situations in which the DBS is the only material available [31]. Our data, even if obtained in a limited number of samples, showed a reduction in the sensitivity of DBS for the detection of NPC, particularly in which the DBS values are in the upper range of the unaffected populations. Correlation studies for LysoSM and LysoSM509 between the plasma and the DBS also underlined the absence of commutability between the two matrixes. As reported by two different studies, the most reliable biomarkers for NPC diagnosis in

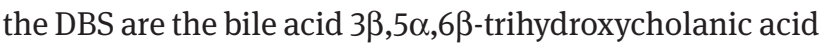
and its glycine conjugate [32, 33].

HexSPh and LysoGb3 measurements have been proposed as biomarkers for monitoring enzyme replacement therapy (ERT) for with FD and GD, respectively [14, 15, 34, 35]. For GD, a new oral drug has become available, thus reducing the number of hospital visits $[36,37]$. The DBS could be useful for the home monitoring of these patients. Furthermore, the patients could collect blood sample by a simple fingerpick and mail the DBS to the laboratory for assay, as is the case in other inherited metabolic diseases (e.g. PKU). To assist the clinical interpretation, a conversion of the DBS to the plasma equivalents would be helpful.

The whole blood collected on filter paper represents an easier and simpler alternative for sample collection and could be particularly attractive for physicians located far from tertiary centers. DBS is widely used to test enzyme activity in a number of lysosomal storage diseases. A twotier approach enzymatic assay combined with the LysoSLs dosage could improve the sensitivity and specificity of the diagnostic test. Additionally, DBS testing has been proved to be a quick and practical method for the identification of a range of lysosomal storage disorders in newborn screening [30]. In our center, we screen neonates for several lysosomal disorders (Gaucher, Fabry, MPS I and Pompe diseases). The analysis of LysoSL in DBS was proposed as a second-tier test in presumptive positive and as an alternative to the more expensive and longer genetic test for FD, NPA/B and KD $[8,11,18]$. Preliminary data showed that HexSph in the DBS could be used as a second-tier test for GD newborn screening.

\section{Conclusions}

In summary, this study showed that DBS analysis with LC-MS/MS could be performed to quantify the levels of LysoSLs. DBS is an efficient matrix to screen several sphingolipidoses (FD, GD, KD and prosaposin deficiency) with analytical performances comparable with those of plasma, which remains the reference matrix for LysoSLs determination. For LysoSM and LysoSM509, plasma seemed to be superior to the DBS screening of NPC and NPA/B. Furthermore, the method could be used for therapeutic monitoring or as a second-tier test in newborn screening.

Acknowledgments: This work was supported by the Associazione COMETA-ASMME (Associazione Studio Malattie Metaboliche Ereditarie). The authors would like to thank Dr. Martina Zaninotto for her scientific assistance.

Author contributions: All the authors have accepted responsibility for the entire content of this submitted manuscript and approved submission.

Research funding: None declared. Employment or leadership: None declared. 
Honorarium: None declared.

Competing interests: The funding organization(s) played no role in the study design; in the collection, analysis, and interpretation of data; in the writing of the report; or in the decision to submit the report for publication.

\section{References}

1. Chamoles NA, Blanco M, Gaggioli D. Diagnosis of alpha-Liduronidase deficiency in dried blood spots on filter paper: the possibility of newborn diagnosis. Clin Chem 2001;47:780 LP-1.

2. Reuser AJ, Verheijen FW, Bali D, van Diggelen OP, Germain DP, Hwu WL, et al. The use of dried blood spot samples in the diagnosis of lysosomal storage disorders - current status and perspectives. Mol Genet Metab (Elsevier Inc) 2011;104:144-8.

3. Li Y, Scott CR, Chamoles NA, Ghavami A, Pinto BM, Turecek F, et al. Direct multiplex assay of lysosomal enzymes in dried blood spots for newborn screening. Clin Chem 2004;50:1785-96.

4. Spacil Z, Tatipaka H, Barcenas M, Scott CR, Turecek F, Gelb MH. High-throughput assay of 9 lysosomal enzymes for newborn screening. Clin Chem 2013;59:502-11.

5. Piraud M, Pettazzoni M, Lavoie P, Ruet S, Pagan C, Cheillan $D$, et al. Contribution of tandem mass spectrometry to the diagnosis of lysosomal storage disorders. J Inherit Metab Dis 2018;41:457-77.

6. Aerts JM, Groener JE, Kuiper S, Donker-Koopman WE, Strijland A, Ottenhoff $R$, et al. Elevated globotriaosylsphingosine is a hallmark of Fabry disease. Proc Natl Acad Sci 2008;105:2812-7.

7. Boutin M, Gagnon R, Lavoie P, Auray-Blais C. LC-MS/MS analysis of plasma lyso-Gb3 in Fabry disease. Clin Chim Acta 2012;414:273-80.

8. Johnson B, Mascher H, Mascher D, Legnini E, Hung CY, Dajnoki A, et al. Analysis of lyso-globotriaosylsphingosine in dried blood spots. Ann Lab Med 2013;33:274-8.

9. Gold H, Mirzaian M, Dekker N, Joao Ferraz M, Lugtenburg J, Codée JD, et al. Quantification of globotriaosylsphingosine in plasma and urine of Fabry patients by stable isotope ultraperformance liquid chromatography-tandem mass spectrometry. Clin Chem 2013;59:547-56.

10. Smid BE, van der Tol L, Biegstraaten M, Linthorst GE, Hollak CE, Poorthuis BJ. Plasma globotriaosylsphingosine in relation to phenotypes of Fabry disease. J Med Genet 2015;52:262-8.

11. Chuang W-L, Pacheco J, Zhang XK, Martin MM, Biski CK, Keutzer $J M$, et al. Determination of psychosine concentration in dried blood spots from newborns that were identified via newborn screening to be at risk for Krabbe disease. Clin Chim Acta 2013;419:73-6.

12. Turgeon CT, Orsini JJ, Sanders KA, Magera MJ, Langan TJ, Escolar $\mathrm{ML}$, et al. Measurement of psychosine in dried blood spots - a possible improvement to newborn screening programs for Krabbe disease. J Inherit Metab Dis 2015;38:923-9.

13. Igisu H, Suzuki K. Analysis of galactosylsphingosine (psychosine) in the brain. J Lipid Res 1984;25:1000-6.

14. Dekker N, van Dussen L, Hollak CE, Overkleeft H, Scheij S, Ghauharali K, et al. Elevated plasma glucosylsphingosine in Gaucher disease: relation to phenotype, storage cell markers, and therapeutic response. Blood 2011;118:e118-27.
15. Rolfs A, Giese A-K, Grittner U, Mascher D, Elstein D, Zimran A, et al. Glucosylsphingosine is a highly sensitive and specific biomarker for primary diagnostic and follow-up monitoring in Gaucher disease in a non-Jewish, Caucasian cohort of Gaucher disease patients. PLoS One 2013;8:e79732.

16. Fuller M, Szer J, Stark S, Fletcher JM. Rapid, single-phase extraction of glucosylsphingosine from plasma: A universal screening and monitoring tool. Clin Chim Acta 2015;450:6-10.

17. Mirzaian M, Wisse P, Ferraz MJ, Gold H, Donker-Koopman WE, Verhoek $M$, et al. Mass spectrometric quantification of glucosylsphingosine in plasma and urine of type 1 Gaucher patients using an isotope standard. Blood Cells Mol Dis 2015;54:307-14.

18. Chuang W-L, Pacheco J, Cooper S, McGovern MM, Cox GF, Keutzer J, et al. Lyso-sphingomyelin is elevated in dried blood spots of Niemann-Pick B patients. Mol Genet Metab 2014;111:209-11.

19. Giese A-K, Mascher H, Grittner U, Eichler S, Kramp G, Lukas J, et al. A novel, highly sensitive and specific biomarker for Niemann-Pick type C1 disease. Orphanet J Rare Dis 2015;10:78.

20. Polo G, Burlina AP, Kolamunnage TB, Zampieri M, Dionisi-Vici C, Strisciuglio $P$, et al. Diagnosis of sphingolipidoses: a new simultaneous measurement of lysosphingolipids by LC-MS/MS. Clin Chem Lab Med 2017;55:403-14.

21. Pettazzoni M, Froissart R, Pagan C, Vanier MT, Ruet S, Latour P, et al. LC-MS / MS multiplex analysis of lysosphingolipids in plasma and amniotic fluid: a novel tool for the screening of sphingolipidoses and Niemann-Pick type C disease. PLoS One 2017;12:1-19.

22. Voorink-Moret M, Goorden SM, van Kuilenburg AB, Wijburg FA, Ghauharali-van der Vlugt JM, Beers-Stet FS, et al. Rapid screening for lipid storage disorders using biochemical markers. Expert center data and review of the literature. Mol Genet Metab 2017;123:76-84.

23. Gaspar P, Codée JD, van der Marel G, Overkleeft HS, Aerts JM. Simultaneous quantitation of sphingoid bases by UPLC-ESI-MS/ MS with identical13C-encoded internal standards. Clin Chim Acta 2017;466:178-84.

24. Motta M, Tatti M, Furlan F, Celato A, Di Fruscio G, Polo G, et al. Clinical, biochemical and molecular characterization of prosaposin deficiency. Clin Genet 2016;90:220-9.

25. Smid BE, Van der Tol L, Biegstraaten M, Linthorst GE, Hollak CE, Poorthuis BJ. Plasma globotriaosylsphingosine in relation to phenotypes of Fabry disease. J Med Genet 2015;52:262-8.

26. Duro G, Zizzo C, Cammarata G, Burlina A, Burlina A, Polo G, et al. Mutations in the GLA gene and LysoGb3: is it really AndersonFabry disease? Int J Mol Sci 2018;19:3726.

27. Nowak A, Mechtler T, Kasper DC, Desnick RJ. Correlation of Lyso-Gb3 levels in dried blood spots and sera from patients with classic and later-onset Fabry disease. Mol Genet Metab 2017;121:320-4.

28. Santoro M, Modoni A, Sabatelli M, Madia F, Piemonte F, Tozzi $\mathrm{G}$, et al. Chronic GM2 gangliosidosis type Sandhoff associated with a novel missense HEXB gene mutation causing a double pathogenic effect. Mol Genet Metab 2007;91:111-4.

29. Welford RW, Garzotti M, Marques Lourenço C, Mengel E, Marquardt T, Reunert J, et al. Plasma lysosphingomyelin demonstrates great potential as a diagnostic biomarker for Niemann-Pick disease type $\mathrm{C}$ in a retrospective study. PLoS One 2014;9:e114669. 
30. Burlina AB, Polo G, Salviati L, Duro G, Zizzo C, Dardis A, et al. Newborn screening for lysosomal storage disorders by tandem mass spectrometry in North East Italy. J Inherit Metab Dis 2018;41:209-19.

31. Kuchar L, Sikora J, Gulinello ME, Poupetova H, Lugowska A, Malinova V, et al. Quantitation of plasmatic lysosphingomyelin and lysosphingomyelin-509 for differential screening of Niemann-Pick A/B and C diseases. Anal Biochem 2017;525:73-7.

32. Jiang X, Sidhu R, Mydock-McGrane L, Hsu F, Covey DF, Scherrer $D E$, et al. Development of a bile acid-based newborn screen for Niemann-Pick disease type C. Sci Transl Med 2016;8:337-63.

33. Mazzacuva F, Mills P, Mills K, Camuzeaux S, Gissen P, Nicoli $E R$, et al. Identification of novel bile acids as biomarkers for the early diagnosis of Niemann-Pick C disease. FEBS Lett 2016;590:1651-62.

34. Arends M, Wijburg FA, Wanner C, Vaz FM, van Kuilenburg $A B$, Hughes DA, et al. Favourable effect of early versus late start of enzyme replacement therapy on plasma globotriaosylsphingosine levels in men with classical Fabry disease. Mol Genet Metab 2017;121:157-61.

35. van Breemen MJ, Rombach SM, Dekker N, Poorthuis BJ, Linthorst $\mathrm{GE}$, Zwinderman AH, et al. Reduction of elevated plasma globotriaosylsphingosine in patients with classic Fabry disease following enzyme replacement therapy. Biochim Biophys Acta Mol Basis Dis 2011;1812:70-6

36. Smid BE, Ferraz MJ, Verhoek M, Mirzaian M, Wisse P, Overkleeft $\mathrm{HS}$, et al. Biochemical response to substrate reduction therapy versus enzyme replacement therapy in Gaucher disease type 1 patients. Orphanet J Rare Dis 2016;11:28.

37. Mistry PK, Lukina E, Ben Turkia H, Shankar SP, Baris H, Ghosn $M$, et al. Outcomes after 18 months of eliglustat therapy in treatment-naïve adults with Gaucher disease type 1: the phase 3 ENGAGE trial. Am J Hematol 2017;92:1170-6.

Supplementary Material: The online version of this article offers supplementary material (https://doi.org/10.1515/cclm-2018-1301). 